\title{
An Alternative Approach to Estimate the Vacuum Energy Density of Free Space
}

\author{
Vernon Cooray ${ }^{1, *}$, Gerald Cooray ${ }^{2}$ and Farhad Rachidi ${ }^{3}$ \\ 1 Department of Engineering Sciences, Uppsala University, Uppsala, Sweden \\ 2 Karolinska Institute, Stockholm, Sweden; gerald.cooray@ki.se \\ 3 École polytechnique fédérale de Lausanne, Lausanne, Switzerland; farhad.rachidi@epfl.ch \\ * Correspondence: vernon.cooray@angstrom.uu.se
}

\begin{abstract}
According to the current understanding, the recently observed accelerated expansion of the universe is caused by the dark or the vacuum energy. Attempts to calculate the magnitude of this energy using the standard model of particle physics led to values which are 59-120 orders of magnitude larger than the experimentally estimated one. Even though the expanding space has positive internal energy, in a flat universe it is completely balanced by the negative energy of gravitational field making the net energy equal to zero. However, the current physical theories may breakdown for times less than or on the order of Planck time and one cannot assume that the above assertion concerning the balance of two energies is valid also in this time scale. In this note it is assumed that this balance of the two energies during the creation of new space as the universe expands takes place only for times larger than the Planck time. If this assumption is correct, the net energy of the newly created space remains positive for times on the order of Planck time and the positive vacuum energy has to be burrowed from empty space before it is being balanced by gravity. This can happen only within the restrictions of the time-energy uncertainty principle. In this note it is shown that such considerations lead to a vacuum energy density of about 0.3 Nanojoules per cubic meter which has to be compared with the measured value of 0.6 Nanojoules per cubic meter.
\end{abstract}

Keywords: expansion of the universe; vacuum energy; dark energy; time energy uncertainty principle; radius of the universe

\section{Introduction}

Recently observed accelerated expansion of the universe can be explained by invoking the concept of vacuum energy which exerts a negative pressure on space causing it to expand $[1,2,3,4,5,6]$. The measured rate of expansion of the universe set the magnitude of this energy to a value close to $6 \times 10^{-10}$ $\mathrm{J} / \mathrm{m}^{3}$ [7]. This energy is believed to be generated by the quantum fluctuations, which leads to the continuous creation and annihilation of virtual particles out of the vacuum [8]. Attempts to derive the magnitude of vacuum energy by appealing to the standard model of particle physics led to values which are 59 to 123 orders of magnitude bigger than the observed value $[9,10]$. It is known as the vacuum catastrophe. The goal of this note is to show that an estimate of the vacuum energy close to the measured ones can be obtained by the application of the time-energy uncertainty principle to the creation of internal energy in the expanding space.

\section{Physical consideration}

As the universe expands, space is continuously created and this newly created space contains internal energy. However, in a flat universe this positive internal energy is balanced by the negative gravitational energy making the net energy gain equal to zero [11]. This in turn means that the net 
energy of the expanding universe remains zero. But, what is the time scale at which this balancing act takes place? There is consensus among the physicists that the physical theories and the notion of space and time will break down in the Planck time scale [5]. In this time scale, the predictions of quantum field theory and general relativity are no longer reconcilable. Guided by this fact, in this note we assume that newly created vacuum energy is balanced by gravitational energy only for times larger than the time $t_{P}$ measured from the time of creation of new space, where the physics as we know it is active. The time $t_{P}$ is on the order of the Planck time. This also means that for times less than about $t_{P}$, the newly created vacuum adds net positive energy to the universe and this net energy, as in the case of the creation of matter in quantum fluctuations, has to be extracted from empty space before it is being 'neutralized' by the negative gravitational energy. The amount of vacuum energy that can be extracted over a given time interval is dictated by the time-energy uncertainty principle. That is the vacuum energy generated within about Planck time, say $\Delta U$, should satisfy the condition $t_{P} \Delta U \square h$. However, there is no general consensus among the physicists that the net energy of the universe is zero. Some physicists believe that the concept of energy conservation is not applicable to the universe as a whole and the vacuum energy adds to the net energy of the universe. In such a scenario, we are assuming that the vacuum energy gained by the expanding universe during each Planck time unit is constrained by the time-energy uncertainty principle. In the analysis to follow, we will show that such considerations lead to values of vacuum energy which are of the same order of magnitude as the measured values.

\section{Mathematical analysis}

The expansion rate of the universe varies with time. This rate was higher at the early stages of the universe in comparison to the present time. However, experiments show that the rate of expansion has started to increase again, roughly about 5 billion or so years ago. Let us denote the radius of the universe in meters by $R$. The rate of change of the radius of the universe $d R / d t$ is denoted by $\Sigma$. With these definitions the amount of new space created within time $t_{P}$ is given by $4 \pi R^{2} \Sigma t_{P}$. Denoting the vacuum energy per unit volume of this newly created space by $\Lambda$, the total vacuum energy generated over the time interval $t_{P}$ is given by $4 \pi R^{2} \Sigma t_{P} \Lambda$. As mentioned in the previous section, this energy has to be burrowed within time $t_{P}$ from empty space and its magnitude is restricted by the time-energy uncertainty principle. That is

$4 \pi R^{2} \Sigma t_{P}^{2} \Lambda \approx h$

Observe that the left hand side of Equation (1) is the product of the vacuum energy and the time over which it is generated. From Equation (1) the vacuum energy density of free space can be obtained as

$\Lambda \approx h / 4 \pi R^{2} \Sigma t_{P}^{2}$

Observe from this equation that the vacuum energy density of newly created space varies inversely with the square of the radius of the universe at that time. As the universe expands, the vacuum energy density associated with newly created space decreases. However, the parameter that is important when analysing the dynamics of the universe using Friedmann equation is the average vacuum energy density of all space corresponding to a given radius of the universe, i.e. average energy of space created from time zero to present time. Let us denote the average vacuum density of all space corresponding to a given radius of the universe by $\Lambda_{\text {ave }}$. Assuming $R \approx k t^{2 / 3}$ as a crude representation of the growth of the actual universe (which is exact for the matter dominated era of the universe), where $k$ is a constant 
and $t$ is the age of the universe, one can derive how the parameter $\Sigma$ varies as a function of $R$. Once this is obtained, the average vacuum energy density corresponding to any given radius of the universe can be obtained easily by solving the following volume integral:

$$
\Lambda_{\text {ave }}=\frac{\int_{r_{0}}^{R} \Lambda 4 \pi r^{2} d r}{\frac{4}{3} \pi R^{3}}
$$

In the above equation $r_{0}$ is the initial radius of the universe. Substituting for $\Lambda$ from (2) and performing the integral and assuming that $R \square r_{0}$, we obtain

$$
\Lambda_{\text {ave }}=\frac{3 h t_{u}}{4 \pi t_{p}^{2} R^{3 / 2} R_{u}^{3 / 2}}
$$

In the above equation $t_{u}$ denotes the current age and $R_{u}$ denotes the current radius of the universe. Observe that the average vacuum energy density of space decreases inversely as the $3 / 2$ root of the radius of the universe. The possibility that the vacuum energy density could vary as the inverse of the square of the radius of the universe was previously suggested by Chen and $\mathrm{Wu}$ [12]. The average vacuum energy at the present time can be obtained by replacing $R$ in the above equation by $R_{u}$. That is

$$
\Lambda_{\text {ave }}=\frac{3 h t_{u}}{16 \pi^{2} t_{p}^{2} R_{u}^{3}}
$$

The current age of the universe (i.e. $\left.t_{u}\right)$ is $4.3 \times 10^{17} \mathrm{~s}$ (13.7 billion years) and the current size of the universe (i.e. $R_{u}$ ) is $4.4 \times 10^{26} \mathrm{~m}$. Substituting these numbers in Equation (6) and together with the values of known constants we find that $\Lambda_{R_{u}}=3 \times 10^{-10} \mathrm{~J} / \mathrm{m}^{3}$. This is a good fit to the experimentally observed value of $6 \times 10^{-10} \mathrm{~J} / \mathrm{m}^{3}$. This shows that the assumption that the positive vacuum energy of the newly created space can be balanced by the negative gravitational energy only for times larger than about the Planck time, and within this time this net energy is constrained by the time-energy uncertainty principle leads to a correct estimate for the vacuum energy density.

As mentioned earlier, the calculated average vacuum energy of space decreases inversely as the $3 / 2$ root of the radius of the universe. The current understanding is that the vacuum energy density of space is a constant and that it does not vary with the age of the universe. However, it is of interest to show that there is another solution where $\Lambda_{\text {ave }}$ becomes independent of the radius of the universe. That is when the quantity $R^{2} \Sigma t_{P}^{2}$ does not vary as the universe age. Let us assume that this quantity is independent of the evolution of the universe. Observing that $t_{P}^{2}$ is proportional to $G / c^{5}$, where $G$ is the gravitational constant and $c$ is the speed of light, and $\Sigma=d R / d t$, the above implies that $R^{2}(d R / d t) G / c^{5}$ is a constant. Assuming $R \square t^{2 / 3}$ as a crude representation of the growth of the actual universe, where $t$ is the age of the universe, we find that $G / c^{5} \square 1 / t$. If the speed of light does not vary with the age of the universe, we obtain $G \square 1 / t$. That is, the gravitational constant decreases inversely as the age of the universe. This is the large number hypothesis of Dirac [13]. 


\section{Discussion}

The main assumption we have made in the derivation is that the total energy of a newly created volume of space remains positive for a time on the order of Planck time before the negative gravitational energy acts on the space volume and make the total energy zero. We have not shown that this is the case by appealing to fundamental physics. However, the fact that this assumption leads to a correct estimate of the vacuum energy calls for a thorough analysis of the energy balance of newly created space volumes. Such an analysis can only be done using a theory of quantum gravity. However, if the assumption that the balance of vacuum energy and gravitational energy takes place only for times larger than the Planck time is correct, it is the time-energy uncertainty principle that acts as the engine in generating the initial conditions necessary for the creation of vacuum energy and hence to the expansion of the universe. Indeed, it has been even suggested that the initial primordial matter (energy) necessary for the creation of the universe also came into being thanks to the time-energy uncertainty principle [14].

The analysis presented here predicts that the average vacuum energy density of the universe varies as the inverse $3 / 2$ root of the radius of the universe. On the other hand, as the universe expands, the matter density will decrease as the inverse cube of the radius of the universe. That means as one goes back towards the beginning of the universe, the matter density increases much faster than the average vacuum energy density. An important time in history is the time or the age of the universe when the vacuum energy density became equal to the matter energy density. This is the time at which the current accelerated expansion of the universe would have started. The current observed value of the ratio of vacuum energy density to the total energy density (both vacuum energy and matter), $\Omega_{\Lambda}$, is about 0.7 [15]. The equations given above show that the two energy densities became equal (observe that the matter energy density decreases as $1 / R^{3}$ while the vacuum energy density decreases as $1 / R^{1.5}$ ) when the radius of the universe was about 0.56 of the current radius. According to the observation the accelerated expansion of the universe started when the size of the universe was about 0.6 of the current value (i.e. redshift 0.55) [15]. This also agrees remarkably well with the estimated value.

One problem associated with the analysis presented in this note that needs further analysis is the following. In deriving Equation (1) and (2), we have treated the energy associated with the newly created space within a time interval $t_{P}$ as a single entity even though this energy is distributed throughout the universe. This may be correct if the newly created space within a Planck time inside the universe is in some way quantum mechanically entangled. This is an interesting problem that needs further attention.

\section{Conclusion}

Based on the assumption that the positive vacuum energy of the newly created space can be balanced by negative gravitational energy only for times larger than about the Planck time, and that within this time this net energy is constrained by the time-energy uncertainty principle we estimated the vacuum energy density of free space. The estimated value is in agreement with experimental observations.

\section{References}

1. Scientific Background on the Nobel Prize in Physics 2011-The accelerating Universe, Royal Swedish academy of Sciences, 2011

2. A.G. Riess et al., Observational evidence from supernovae for an accelerating universe and a cosmological constant", Astron. J., 116, 1009-1038, 1998. 
3. S. Perlmutter et al., Measurement of $\Omega$ and $\Lambda$ from 42 high-redshift supernovae, Astrophys. J., 517, 565-586, 1999.

4. Pasachoff, J. M. and A. Filippenko, Astronomy in the new millennium, 1st edition, Brooks/Cole, 2001.

5. Hawking, S., The theory of everything, New Millennium, 2002.

6. https://science.nasa.gov/astrophysics/focus-areas/what-is-dark-energy

7. Komatsu, E. et al., Seven-Year Wilkinson Microwave Anisotropy Probe (WMAP) Observations: Cosmological Interpretation, Astrophys. J. Suppl.192, 18, 2011.

8. https://en.wikipedia.org/wiki/Vacuum_energy

9. Milonni, P. W, The Quantum Vacuum: An Introduction to Quantum Electrodynamics. Academic Press, Boston, 1994.

10. De la Peña, Luis, A. M. Cetto ,The Quantum Dice-An Introduction to Stochastic Electrodynamics, Springer, 1996.

11. Edward P. Tryon, Is the Universe a Vacuum Fluctuation?, Nature, vol. 246, p.396-397, 1973.

12. Chen, W. and Y-S Wu, Implication of a cosmological constant varying as $\mathrm{R}^{-2}$, Physical Review D, 41, $695-698,1990$.

13. Dirac, P. A. M., The large numbers hypothesis and Einstein's theory of gravity, Proc. R. Soc. Lond. A. $365,19-30,1979$.

14. Dongshan He, Dongfeng Gao, Qing-yu Cai, Spontaneous Creation Of The Universe From Nothing, arXiv: 1404.1207v1, 2014.

15. Velten, H. E. S., R. F. vom Marttens, and W. Zimdahl, Aspects of the cosmological "coincidence problem", The European Physical Journal C, 74:3160, 2014. 